\title{
Towards Modern Air-to-Air Communications: the LDACS A2A Mode
}

\author{
Miguel A. Bellido-Manganell \\ Institute of Communications and Navigation \\ German Aerospace Center (DLR) \\ Wessling, Germany \\ Miguel.BellidoManganell@DLR.de
}

\author{
Michael Schnell \\ Institute of Communications and Navigation \\ German Aerospace Center (DLR) \\ Wessling, Germany \\ Michael.Schnell@DLR.de
}

\begin{abstract}
The increasing air traffic foreseen for the next decades has triggered an extensive modernization of the air traffic management. Specifically, new air traffic services and operational concepts have been defined and shall be supported during all phases of flight by a set of modern digital data links integrated into a single communications network named the Future Communications Infrastructure (FCI). The air-to-air (A2A) component of the FCI, the L-band Digital Aeronautical Communications System (LDACS) A2A mode, is currently in the initial stages of its development. Given that the LDACS A2A mode must be able to operate without any ground or satellite support, the data link must provide means for the aircraft to establish and organize an independent communications ad-hoc network, which imposes a great challenge for the design of the data link and specially for its medium-access control. In this paper, we contribute to the development of the LDACS A2A mode by assessing the performance of an A2A data link based on two different mediumaccess protocols; ALOHA with and without diversity, and a self-organizing time-division multiple-access (STDMA) scheme. The performance is obtained by simulating the implemented models of both the ALOHA-based and the STDMA-based A2A data link for different design parameters, requirements, and air traffic conditions. The obtained results show that the STDMAbased A2A data link performs better than the ALOHA-based A2A data link in most considered cases, given than the former requires a lower bandwidth than the latter to achieve the desired performance. Based on our analysis, we conclude that STDMA is a better candidate than ALOHA with or without diversity for the medium-access control of the LDACS A2A mode.
\end{abstract}

Index Terms-AANET, air-to-air, aircraft-to-aircraft, A2A, A/A, ALOHA, FANET, LDACS, medium-access control (MAC), self-organizing, STDMA, TDMA.

\section{INTRODUCTION}

In order to sustain the continuous air traffic growth expected for the next decades, new services and operational concepts for air traffic management have been defined in [1]. These are to be supported by the Future Communications Infrastructure (FCI), a set of communication data links developed to jointly cover all phases of flight. Whilst communications in oceanic and remote regions are covered by satellite communication systems, communications on airports are supported by the Aeronautical Mobile Airport Communications System (AeroMACS). In addition, the L-band Digital Aeronautical Communications System (LDACS) completes the FCI by supporting both air-ground $(\mathrm{A} / \mathrm{G})$ and air-to-air (A2A) communications using the LDACS A/G and LDACS A2A communication modes, respectively. Whereas the LDACS A/G mode is already fully developed, has been demonstrated in flights trials in March/April 2019 in the MICONAV project [2], and is currently undergoing the standardization process of the International Civil Aviation Organization (ICAO), the LDACS A2A mode is currently being developed within the German national project IntAirNet.

There are some challenges for the design of the LDACS A2A mode. First, the scarcity of free spectrum in the Lband, where LDACS operates, significantly limits the design freedom with respect to the radiated power, suitable frequency allocations, and usable spectrum bandwidth. Second, in contrast to the LDACS A/G, the LDACS A2A must be able to operate without any external support, given that it must also support aircraft-to-aircraft communications in oceanic, remote, and polar (ORP) regions, and in autonomous operation areas [1], where support from satellites or ground infrastructure might not be available. Consequently, the LDACS A2A mode must provide means for the aircraft to establish and organize a communications ad-hoc network without any external support. Such a network entails numerous additional challenges for the design, primarily in the medium-access control and the network routing. To enable the new services and operational concepts [1], the LDACS A2A mode shall support broadcast communications, for concepts such as self-separation and wake vortex prediction, and point-to-point communications to allow aircraft to negotiate trajectories, resolve conflicts, and use other aircraft as relays to enable communications beyond radio line-of-sight.

In this paper, we contribute to the development of the LDACS A2A mode by focusing on the broadcast component of the link. In this respect, we assess the performance of an A2A data link based on two well-known medium-access protocols that might be employed for the LDACS A2A mode; ALOHA with and without diversity, and a self-organizing time-division multiple-access (STDMA) scheme. The performance is obtained by simulating an A2A data link model based on these medium-access protocols under the dense air traffic expected for the future. Based on the obtained results, we discuss some of the key parameters for the design of the LDACS A2A mode, such as the minimum bandwidth that will have to be employed in each considered scenario, or 
the effect of designing the link for a longer communications range or transmitting more information bits per broadcast message. Finally, we compare both medium-access protocols and discuss their suitability for the LDACS A2A mode.

\section{BACKGROUND ON LDACS}

As a detailed description of LDACS can be found in [3], [4], we only discuss the main aspects of the current LDACS specification, i.e., the LDACS A/G mode, that play a role in our analysis.

LDACS is a cell-based aeronautical communications system operating in the frequency band $960-1164 \mathrm{MHz}$ and supporting data and voice communications between ground stations and airborne stations. An LDACS ground station is located in the center of each cell and communicates with the LDACS airborne stations located in the aircraft flying within its cell. Using a frequency-division duplex scheme, the LDACS ground station transmits in the forward link (FL) of the cell at the same time as the airborne stations transmit in its reverse link (RL). These transmissions in the RL are structured in tiles with a duration of $0.72 \mathrm{~ms}$ and occupying half of the channel bandwidth per tile, i.e., two tiles are transmitted simultaneously in the RL. The ground station allocates the resources of the RL, i.e., the available tiles, to the registered aircraft under demand. Using an adaptive coding and modulation scheme, each tile can carry at least 112 information bits using the 134 available complex symbols.

Note that a ground station could also organize the resources in the A2A link in a similar way as it is done in the RL. However, as the A2A link must be capable of operating in regions without any ground or satellite support, a standalone operation mode is required and is the focus of this paper. Nevertheless, the A2A link should be designed in such a way that the eventual support from a ground station, if available, can be used to improve the performance of the A2A link.

Both the FL and the RL of a cell use a frequency channel of $0.5 \mathrm{MHz}$ each. Because of the scarcity of free spectrum in the L-band, LDACS is expected to be deployed following an inlay approach, where each $0.5 \mathrm{MHz}$ channel is located between two channels of the distance measuring equipment (DME) spaced $1 \mathrm{MHz}$ apart. This point is specially important for our analysis, given that it indicates that using a bandwidth higher than $0.5 \mathrm{MHz}$ for the A2A link might only be possible if it is split into sub-channels of $0.5 \mathrm{MHz}$.

\section{Medium-Access Control Schemes}

In any wireless communications system, messages propagate from the transmitter to the receiver through a wireless channel. If the channel conditions are favourable and the received signal power is high enough, the receiver should be able to correctly decode the message. Nevertheless, if several users want to transmit messages and no coordination takes place beforehand, their transmissions might collide at the receiver and interfere each other, consequently hindering or even preventing the successful decoding of the messages. In general, whilst coordinated medium-access schemes try to avoid harmful collisions by coordinating the transmissions beforehand, uncoordinated or contention-based medium-access schemes rely on the probability of the message to be delivered successfully, despite the possible collisions.

In this work, we consider two radically different mediumaccess methods for our analysis; an asynchronous and uncoordinated ALOHA medium-access scheme, and a synchronous and coordinated STDMA scheme.

\section{A. ALOHA}

Initially proposed in [5] as a communications protocol for a computer network, it has been employed in many communication systems because of its simplicity and effectiveness.

In the ALOHA protocol, users transmit their messages in an asynchronous and uncoordinated way and rely on the probability of the message being eventually received successfully, despite collisions with other transmissions might occur.

From the initial ALOHA proposal, known as pure ALOHA, numerous variants have been employed over the years, such as slotted ALOHA [6], diversity ALOHA [7], spread spectrum ALOHA [8], or ALOHA with interference cancellation [18]. We focus our analysis on pure ALOHA given that it is used by several air-to-air aeronautical communication systems such as the $1090 \mathrm{MHz}$ (Mode S) Extended Squitter (1090ES) [9]. Moreover, its asynchronous operation makes it specially interesting for safety-of-life applications, given that it would not depend on a common timing source. We additionally consider ALOHA with diversity, where multiple replicas of the same message are transmitted in order to increase the probability of correctly receiving the message.

\section{B. Self-Organizing Time-Division Multiple-Access Scheme}

In a time-division multiple-access scheme, transmissions are bound to time slots of fixed duration, which are commonly organized into frames. Assuming that a common scheduling has been agreed and that there are more time slots than users, all users should be able to exclusively transmit their messages in one or several time slots, avoiding consequently any collision. However, a common scheduling is not always easy to achieve, given that the transmissions from all users in the network must be taken into account. In the absence of a central entity capable of performing and conveying the common scheduling, e.g., a ground-station, users must coordinate their transmissions in a different way. Although numerous possibilities to reach a common scheduling have been proposed in the literature, we focus on STDMA, which was initially proposed in [10] and is currently employed by the shipborne automatic identification system (AIS) [11] for ship-to-ship communications and is part of the VHF Digital Link (VDL) Mode 4 [12] for aircraft-toaircraft communications.

In STDMA, when a user transmits a message, it additionally indicates the time $\operatorname{slot}(\mathrm{s})$ that will be used next. Users receiving and decoding the message will then choose a different time slot for their transmissions, ideally avoiding any collision. Reservation can be made implicitly, for example by always using the same time slot in each frame, or explicitly. An 
example of the operation of STDMA with explicit reservations is shown in Fig. 1, where the users A and B transmit messages respectively in the first and the second time slots of the $n$-th frame. In addition to the payload information transmitted in the messages, these also indicate explicitly that the users A and $\mathrm{B}$ will use respectively the $p$-th and the third time slots of the next frame. Consequently, the user $\mathrm{C}$, who has received and successfully decoded the transmissions from the other users, knows that the second time slot of the next frame will now be free and transmits its message in it. As shown in Fig. 1, the duration of the time slot must be higher than the duration of a message, given that a propagation guard must be left after each transmission for the message to reach the intended receivers without collisions.

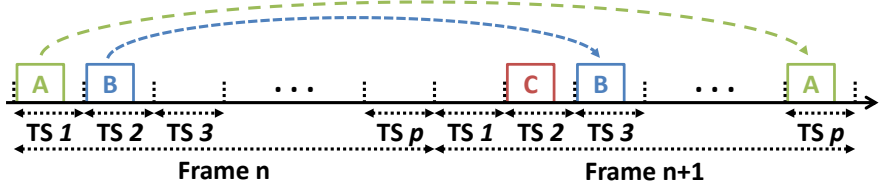

Fig. 1. Example of the operation of STDMA. The horizontal axis represents the time, which is divided into frames and time slots (TSs).

The main advantage of STDMA is that a common scheduling can be reached if all users can correctly decode the transmissions from the other users, without the need of exchanging additional messages to negotiate the scheduling. However, this is not always the case, because some users might be outside the communications range of other users and collisions might occur when they transmit in the same time slot. In the example shown in Fig. 1, if users A and B are not capable of decoding the transmissions from each other, both users might use the same time slot in the next frame. This problem, commonly known as the hidden node problem, might lead to a collision at user $\mathrm{C}$, who might not be able to decode any of the received messages. Although solving the hidden node problem is feasible, for example by broadcasting the slot-usage information observed by each user, additional transmissions or overhead would be needed. In our analysis, we employ a basic version of STDMA and consequently we do not consider any countermeasure implying either more overhead or more transmissions to avoid or mitigate the hidden node problem.

Another advantage of STDMA is its inherent suitability to periodic transmissions, e.g., surveillance broadcasts. In addition, its operation could be easily supported by a central entity if needed, e.g., the common scheduling could be performed by an LDACS ground-station in regions with a dense air traffic to improve the performance of the system.

\section{Methodology}

\section{A. Aircraft Distribution}

In order to evaluate the effectiveness of the discussed medium-access schemes, realistic operating conditions have to be considered for the simulations. Given that we focus on the performance of different medium-access schemes, the main parameter to be defined is the number of aircraft that will

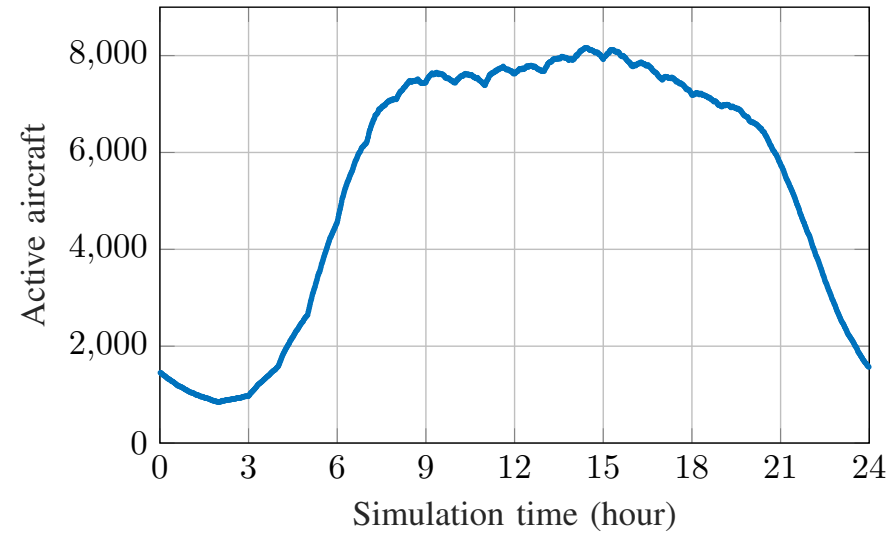

Fig. 2. Number of simultaneously active aircraft in the generated air traffic for one summer day in 2035 using the FACTS2 simulator.

use the A2A data link. Even though the current geographical aircraft distribution could be obtained from one of the available databases, such as FlightRadar24 [13] or the OpenSky Network [14], it would only be useful to evaluate how the medium-access schemes would perform nowadays. However, the main objective of developing a new A2A data link is to sustain the continuous air traffic growth and, consequently, its performance should be assessed under the operating conditions that will have to be faced in the future. Consequently, we employ in our analysis the FACTS2 simulator described in [15], which can simulate future pan-European air traffic by modelling the probability distribution of flights between airports as Poisson processes and adjusting the model based on real flight plans and the predicted air traffic growth.

Using the FACTS2 simulator, we generate pan-European air traffic on a busy summer day of $2035^{1}$, with up to almost 49,000 flights taking place along the day [17]. As shown in Fig. 2, the number of simultaneously active aircraft fluctuates strongly and reaches a maximum of 8,161 active aircraft shortly after 14:00.

More interesting for the A2A data link is the geographical distribution of aircraft, as it indicates the number of aircraft that will have to be supported in each region. Throughout the entire simulation, the highest aircraft concentration is found in the area comprising South East Great Britain, North East France, Belgium, the Netherlands, and West Germany, which can be seen in the snapshot of the aircraft positions depicted in Fig. 3 for a time instant of the simulation.

For the evaluation of the discussed medium-access schemes, we derive some statistics from the simulated air traffic. As one of the most important parameters is the number of aircraft within communications range and radio line-of-sight, we obtain the number of aircraft within several ranges for each instant and aircraft. The maximum number of aircraft within each range is depicted in Table I, together with the considered range around the aircraft and the maximum and 95th percentile

\footnotetext{
${ }^{1}$ We consider the scenario A of the FACTS 2 simulator, which accurately estimates the air traffic evolution as verified in [16].
} 


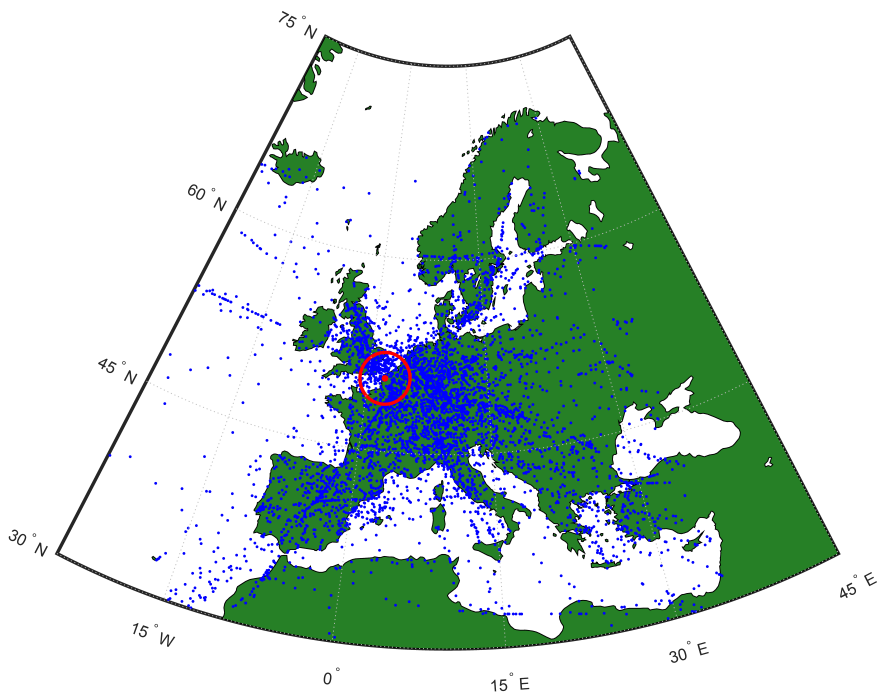

Fig. 3. Snapshot of the aircraft positions (blue dots) at the time instant of the FACTS2 simulation when the maximum number of aircraft were observed within a radius of 120 nautical miles (NM). The red dot represents the central aircraft seeing the maximum number of aircraft, i.e., 771, within the $120 \mathrm{NM}$ radius depicted with the red circle.

aircraft density perceived around each aircraft.

TABLE I

MAXIMUM AIRCRAFT AND OBSERVED AIRCRAFT DENSITIES WITHIN EACH CONSIDERED RANGE.

\begin{tabular}{c|c|c|c}
$\begin{array}{c}\text { Range } \\
(\mathbf{N M})\end{array}$ & $\begin{array}{c}\text { Maximum } \\
\text { aircraft in } \\
\text { range }\end{array}$ & $\begin{array}{c}\text { Maximum } \\
\text { aircraft density } \\
\text { (aircraft/NM }\end{array}$ & $\begin{array}{c}\text { 95th percentile } \\
\text { aircraft density } \\
\text { (aircraft/NM }\end{array}$ \\
\hline 120 & 771 & 0.0170 & 0.0131 \\
150 & 1131 & 0.0160 & 0.0117 \\
200 & 1604 & 0.0128 & 0.0110 \\
500 & 4342 & 0.0055 & 0.0054
\end{tabular}

Additionally, the fluctuating aircraft density can be observed in Fig. 4, where the aircraft density observed in a range of 500 NM around each point in space is shown for the time instant when the maximum number of aircraft within that range was measured throughout the simulation. As it can be seen, a single aircraft density value cannot be deduced from the results, given that some regions show a very high aircraft density whilst other regions, such as the entrance to the North Atlantic Corridor, exhibit a low concentration of aircraft.

In order to evaluate the discussed medium-access schemes through simulations, we consider an aircraft distribution model based on the observed statistics of the generated air traffic. First, we consider that aircraft are uniformly distributed over a 2-D plane with a constant aircraft density $\rho$. We use two values of $\rho$ in our analysis to model the air traffic. Initially, we consider $\rho=0.0055$ aircraft $/ \mathrm{NM}^{2}$ to recreate the scenario where the maximum number of aircraft within a $500 \mathrm{NM}$ radius has been measured, as shown in Table I. Therefore, $\rho=0.0055$ aircraft $/ \mathrm{NM}^{2}$ is considered to be a realistic aircraft density, which however might not accurately represent the presence of localized high aircraft densities. Consequently,

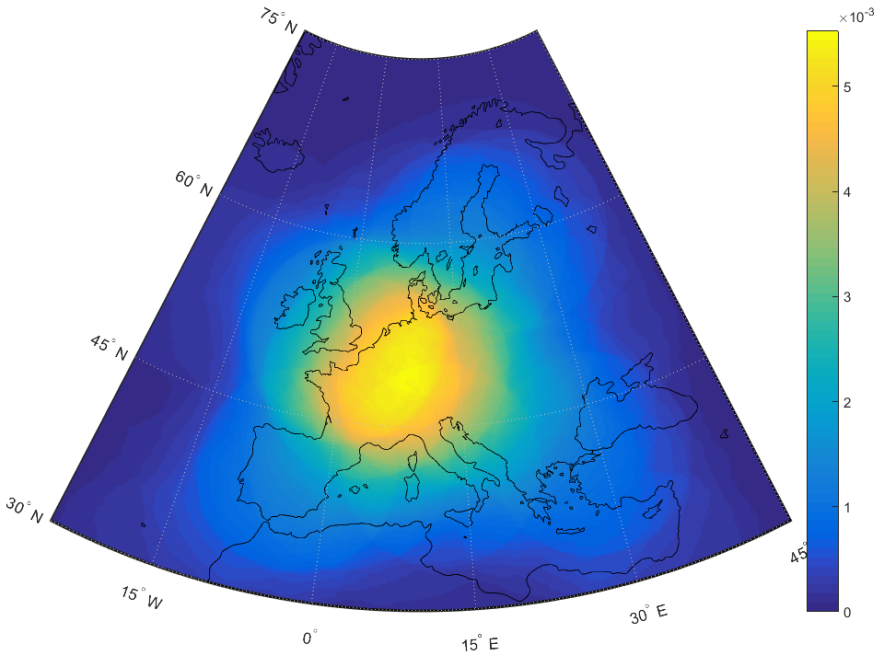

Fig. 4. Observed aircraft density (aircraft/NM ${ }^{2}$ ) in a $500 \mathrm{NM}$ radius around each point of space at the time instant when the maximum aircraft density was measured.

we also use $\rho=0.015$ aircraft $/ \mathrm{NM}^{2}$ to represent very dense airspaces. Nevertheless, this is a very conservative assumption because we consider a constant aircraft density for the entire airspace and not only for the scattered regions where such a high density has been observed. Second, we model the earth curvature by assuming a maximum radio line-of-sight, i.e., the range up to which a signal will propagate before been blocked by the earth curvature, of $R_{l o s}=500 \mathrm{NM}$, which is slightly higher than the maximum radio line-of-sight of two aircraft flying at a height of $12 \mathrm{~km}$ over the ground, which is approximately $488 \mathrm{NM}$. Consequently, we assume in our simulations that aircraft receive the transmissions of other aircraft up to a distance of $R_{\text {los }}=500 \mathrm{NM}$. Note that this is a conservative assumption leading to a stronger background interference, given that the communications range of the system, $R_{c}$, will be significantly lower than $R_{l o s}$.

\section{B. A2A Data Link Model}

In the implemented model of the A2A data link, each aircraft transmits one message containing $L$ information bits every $T$ seconds on average, using a total bandwidth $B W$ split into $N_{s c}$ frequency sub-channels. We use a transmit power such that the signal-to-noise ratio (SNR) at the desired communications range $R_{c}$ is $S N R_{c}$ in the absence of interference, as it is commonly considered in similar studies, e.g., in [18], [19]. As the A2A data link will also be used for surveillance, we assume that each broadcast message contains the position of the transmitter, its identification, and other parameters.

As we expect a physical layer similar to the one employed in the LDACS RL, we assume the same spectral efficiency to the one achieved by the LDACS RL, i.e., data are carried by the 134 complex symbols transmitted in each tile with a duration of $0.72 \mathrm{~ms}$ and occupying $0.25 \mathrm{MHz}$. Consequently, 
each transmitted message has a duration

$$
T_{m}=\frac{L / a}{B W / N_{s c}} \cdot \frac{0.25 \cdot 10^{6} \cdot 0.72 \cdot 10^{-3}}{134} \mathrm{~s},
$$

where $a$ represents the rate of the coding and modulation scheme, such that $L$ information bits are transmitted using $L / a$ complex symbols.

When a transmitted message is received by an aircraft at a distance $d$ of the transmitter, the signal-to-interference-andnoise ratio (SINR) is computed as

$$
S I N R=\frac{d^{-\alpha}}{\frac{R_{c}^{-\alpha}}{S N R_{c}}+I},
$$

where a Gaussian joint interference component $I$ is assumed and $\alpha$ represents the free-space path-loss exponent, i.e., $\alpha=2$.

The joint interference component $I$ is caused by the transmissions from other aircraft that are received simultaneously with the desired message. This is obtained in our analysis as

$$
I=\sum_{i=1}^{N_{I}} \tau_{i} \cdot d_{i}^{-\alpha}
$$

where $N_{I}$ is the number of interferers, $d_{i}$ represents the distance from the receiver to each one of them, and Gaussian interference has been assumed. In addition, $\tau \in[0,1]$ represents the overlapping factor between the desired message and each interfering signal. Consequently, we assume an interference model where the interference power is weighted with the overlapping factor between the desired message and the interference, as it is commonly assumed in similar studies, e.g., in [18].

Once the SINR is obtained for each message using Eq. (2), we employ the following approximation to the minimum achievable packet error rate (PER) [18]

$P \approx \frac{1}{2} \operatorname{erfc}\left(B\left(\log _{2}(1+S I N R)-a+\frac{a}{2 L} \log _{2}\left(\frac{2 L}{a}\right)\right)\right)$,

with

$$
B=\frac{(1+S I N R) \ln (2)}{\sqrt{2 \frac{a}{L} S I N R(2+S I N R)}},
$$

where $\operatorname{erfc}(\cdot)$ represents the complementary error function [20]. Note that we assume that each packet is composed of a single codeword and messages are affected by additive white Gaussian noise (AWGN).

In addition, we consider a half-duplex scheme, where no aircraft can transmit and receive at the same time. Consequently, when an aircraft receives a message while transmitting its own message, the received message is directly considered to be lost, regardless of its SINR or the overlapping factor. This consideration is necessary because the analog-to-digital converter of the receiver is saturated during its own transmissions and consequently no reception is possible under such conditions. Note that this limitation prevails even when multiple frequency sub-channels are considered, as they are all assumed to be located in the same frequency band.
We now discuss the two models used to represent the behaviour of the system when either an ALOHA-based or a STDMA-based A2A data link is used.

1) ALOHA-based A2A Data Link Model: We extend the general model of the A2A data link described in Section IV-B for the case when ALOHA is used as medium-access protocol. During the $T_{m}$ seconds that a desired message is being received from a distance $d$, other messages might interfere the desired message. Given that aircraft transmit asynchronously at an average rate $k / T$, are assumed to be uniformly distributed over the 2-D plane, and use randomly one of the $N_{s c}$ sub-channels with equal probability, the interfering signals can be modelled as a Poisson process with an average number $\lambda$ of interferers per desired message, where

$$
\lambda=\frac{2 k \cdot T_{m}}{T} \frac{\rho \pi R_{l o s}^{2}}{N_{s c}} .
$$

The variable $k$ represents the number of replicas transmitted per each message (in pure ALOHA, $k=1$ ). This has been included in the model because of its simplicity and the possibility of optimizing the performance by properly designing $k$. Note that a message of duration $T_{m}$ is interfered by any other signal received within $T_{m}$ seconds before and after the beginning of the desired reception, which leads to the factor of 2 included in Eq. (6).

For each received message, a realization of the Poisson distribution parametrized by Eq. (6) is obtained, indicating the number of interferers $N_{I}$ to be considered in Eq. (3). In addition, as aircraft are uniformly distributed and transmit asynchronously, each overlapping factor $\tau_{i}$ is obtained as a realization of a uniformly-distributed random variable between 0 and 1, i.e., $\tau \sim U(0,1)$.

The uniform aircraft distribution allows us to obtain the distance $d_{i}$ to each interferer as a realization of a random variable parametrized by the cumulative density function $F\left(d_{i}\right)=\frac{1}{R_{l o s}^{2}} d_{i}^{2}$ defined between 0 and $R_{l o s}$.

Once the interference component $I$ and the SINR are obtained using respectively Eq. (3) and Eq. (2), the PER is estimated using Eq. (4). In order to take into account the outages caused by the receiver transmissions, the probability of interfering a received message with its own transmissions is estimated as

$$
P_{\text {own }} \approx \frac{2 k \cdot T_{m}}{T},
$$

where it is assumed that $T \gg k \cdot T_{m}$, i.e., a very low duty cycle per aircraft, and that at least a gap of $T_{m}$ is left between replicas. Note that, as discussed in Section IV-B, all frequency sub-channels are affected by these outages.

Consequently, the PER for the ALOHA-based A2A data link can be obtained as

$$
P E R_{A L O H A}=P+P_{\text {own }}-P \cdot P_{\text {own }},
$$

where $P$ and $P_{\text {own }}$ are obtained using Eq. (4) and Eq. (7), respectively.

Given that $k$ replicas might be transmitted, the probability of losing a message equals the probability of losing the $k$ replicas 
transmitted per message. Consequently, assuming that replica losses are independent events, the message error rate (MER) of the ALOHA-based A2A data link can be finally obtained as

$$
M E R_{A L O H A}=\left(P E R_{A L O H A}\right)^{k},
$$

using Eq. (8) to obtain $P E R_{A L O H A}$.

2) STDMA-Based A2A Data Link Model: We now extend the general model of the A2A data link described in Section IV-B with the specific characteristics of a STDMAbased A2A data link. In contrast to the semi-analytical model developed for ALOHA, the STDMA model is completely based on simulations, given that no realistic analytical model has been found in the literature.

The considered STDMA-based A2A data link uses time slots of duration $T_{t s}$ and frames of duration $T$, such that each aircraft transmits once per frame. For a nominal communications range $R_{c}$, a propagation guard of at least $\frac{R_{c}}{3 \cdot 10^{8} \mathrm{~m} / \mathrm{s}}$ is left after the transmission of the message of duration $T_{m}$ in each time slot. In order to have an integer number of time slots per frame, the time slot duration is obtained as

$$
T_{t s}=T \cdot\left\lfloor\frac{T}{T_{m}+\frac{R_{c}}{3 \cdot 10^{8} m} s}\right\rfloor^{-1}
$$

and consequently

$$
T_{g}=T_{t s}-T_{m}
$$

considering $T_{t s}>T_{m}$. Note that a higher communications range leads to a longer time slot duration and, consequently, to a smaller set of time slots per frame. In addition, we do not consider the overhead needed for the reservations, which is an optimistic approximation for STDMA, specially for a low $L$.

For each simulation of the implemented STDMA-based A2A data link model, a realization of the aircraft distribution model described in Section IV-A is first obtained for the considered aircraft density $\rho$ in a circle of radius $2 R_{\text {los }}$. Then, each aircraft chooses a slot to transmit its message based on two possible criteria. First, if only one frequency subchannel is used, i.e., if $N_{s c}=1$, the aircraft computes the expected interference in each time slot based on the received reservations and using Eq. (3) with $\tau_{i}=1 \forall i$, and chooses the time slot with the minimum expected interference. If the minimum interference level is found in several time slots, one of them is chosen randomly with equal probability. Second, if several sub-channels are used, i.e., if $N_{s c}>1$, the aircraft tries to avoid outages caused by its own transmissions by relying on the maximum reuse distance of a slot. This is, the aircraft estimates its distance to the closest aircraft intending to use each slot. This distance is known as the reuse distance of the slot. Then, considering the $N_{s c}$ concurrent slots contained in each time slot, the minimum reuse distance of each time slot is obtained. Finally, the time slot with the maximum reuse distance is chosen, as well as the sub-channel with the maximum reuse distance in the chosen time slot. In case that several time slots or slots have the same reuse distance, one of them is chosen randomly with the same probability. Note that more complex algorithms reaching better performances are possible, but the implemented algorithms are considered to be sufficient for the purpose of our study.

Initially, all reservations from aircraft up to a distance of $R_{\text {los }}$ are assumed to be correctly received. Then, when several iterations are simulated, i.e., several consecutive frames, the resulting performance of each iteration is used as the criterion to decide if reservations from aircraft separated a certain distance are received, which consequently affects the scheduling for the next iteration. Simulating more than one iteration realistically represents the behaviour of a STDMA-based A2A data link with memory. However, as each iteration requires an extremely high computational effort, more than one iterations have only been considered for some specific cases explicitly mentioned in Section V.

After all aircraft have scheduled their transmissions, they transmit in the chosen slots. Then, we analyse the reception of each message by each aircraft up to $R_{l o s}$ as follows. First, for each received message transmitted from a distance $d$, the overlapping factors of the interfering messages are obtained, considering a signal propagation velocity of $3 \cdot 10^{8} \mathrm{~m} / \mathrm{s}$. Then, the interference power and the SINR are computed using Eq. (3) and Eq. (2), respectively. We assume that messages received in different sub-channels do not affect each other. However, in case that the own transmission of the receiver overlaps the received message, the SINR is set to 0 for the received message regardless of the used sub-channel. Finally, the MER of the message is obtained using Eq. (4).

After all receptions by all aircraft up to $R_{l o s}$ have been processed, we obtain the MER of the STDMA-based A2A data link for a distance $d$ between a transmitter and a receiver by averaging all receptions that happened at that distance as

$$
\overline{M E R}[d]=\frac{1}{N} \sum_{j: d_{j} \in[d-\Delta d, d+\Delta d]} P_{j},
$$

where $P_{j}$ is the MER of the $j$-th message received from a distance $d_{j}$ obtained using Eq. (4), out of a total of $N$ messages received approximately from a distance $d$. Note that $\Delta d$ represents the resolution in the obtained results. For our results, we consider a resolution of $\Delta d=2.5 \mathrm{NM}$, which has been observed to be sufficiently accurate.

\section{Results}

For the simulations, we employ the parameters shown in Table II.

TABLE II

SIMULATION PARAMETERS.

\begin{tabular}{|c|c|c|c|}
\hline$R_{c}[\mathrm{NM}]$ & $\{120,150,200\}$ & $R_{\text {los }}[\mathrm{NM}]$ & 500 \\
$L[\mathrm{bits}]$ & $\{14,34\} \cdot 8$ & $S N R_{c}[\mathrm{~dB}]$ & 6 \\
$B W[\mathrm{MHz}]$ & {$[0.5: 0.5: 5]$} & $T[\mathrm{~s}]$ & 1 \\
$N_{s c}$ & $\left\{1, \frac{B W}{0.5 \mathrm{MHz}}\right\}$ & $\rho\left[\frac{\text { aircraft }}{\mathrm{NM}^{2}}\right]$ & $\{0.0055,0.0150\}$ \\
\hline
\end{tabular}

We have considered three nominal communication ranges for the evaluation of the A2A data link; $120 \mathrm{NM}, 150 \mathrm{NM}$, 
and $200 \mathrm{NM}$. Whilst a communications range of $120 \mathrm{NM}$ corresponds to the maximum A2A surveillance range desired (but not required) for the automatic dependant surveillance - broadcast (ADS-B) [21], a communications range of 150 NM is the largest fixed-range transmission volume defined for broadcasts in [1] and was used in [18] as a conservative assumption for beaconing, and a maximum communications range of $200 \mathrm{NM}$ has been defined in [1]. Note that the latter is a very conservative assumption since it is only intended for addressed communications [1]. However, we also take it for our analysis given that long-range broadcasts might be needed for other communication procedures, such as routing. Consequently, the three communication ranges are considered in our analysis as they might be used for the LDACS A2A data link.

We initially assume that each message must carry 14 information bytes of data, which equals both an ADS-B broadcast of the $1090 \mathrm{MHz}$ (Mode S) Extended Squitter and the information data transmitted in one tile of the LDACS RL when the most robust coding and modulation scheme is used, as discussed in Section II. We also study the effect of transmitting 34 information bytes of data per message, as it is recommended for broadcasts in [1]. Moreover, we consider that the A2A link might either use a single frequency channel of bandwidth $B W$, i.e., $N_{s c}=1$, or multiple sub-channels of $0.5 \mathrm{MHz}$ each, i.e., $N_{s c}=B W / 0.5 \mathrm{MHz}$, as discussed in Section II. Furthermore, each aircraft broadcasts a message on average every $T=1 \mathrm{~s}$, which equals the minimum update period desired for ADS-B [21]. The SNR at $R_{c}$, which is assumed to be $S N R_{c}=6 \mathrm{~dB}$, is considered to be realistic for a communication range of $120 \mathrm{NM}$ and $150 \mathrm{NM}$ based on the LDACS RL link budget [3], and challenging for $200 \mathrm{NM}$, although it might have to be confirmed in a real deployment.

In addition to assessing the performance of an A2A data link based on either ALOHA or STDMA under realistic and challenging air traffic conditions, we use the implemented model to estimate the minimum bandwidth that either an ALOHA-based or STDMA-based A2A data link will require in order to achieve the minimum desired performance. The minimum required bandwidth is obtained for each combination of the desired communications range $R_{c}$, the information bits $L$ transmitted per message, the number of employed subchannels $N_{s c}$, and the considered aircraft density $\rho$. The design parameters $a$ and $k$ are taken such that the performance is optimized. As the minimum required performance, we define that the A2A link must be able to convey a message within the communications range with a MER lower than $10^{-3}$, as considered in [18]. Given the scarcity of free spectrum in the L-band, we consider a maximum bandwidth of $5 \mathrm{MHz}$.

\section{A. Results for the ALOHA-Based A2A Data link}

We simulate the ALOHA-based A2A data link for the aforementioned parameters for an aircraft density $\rho=0.0055$ aircraft $/ \mathrm{NM}^{2}$ and using a single channel, i.e., $N_{s c}=1$. The bandwidth has been increased for each case from $0.5 \mathrm{MHz}$ in steps of $0.5 \mathrm{MHz}$ until either the desired performance

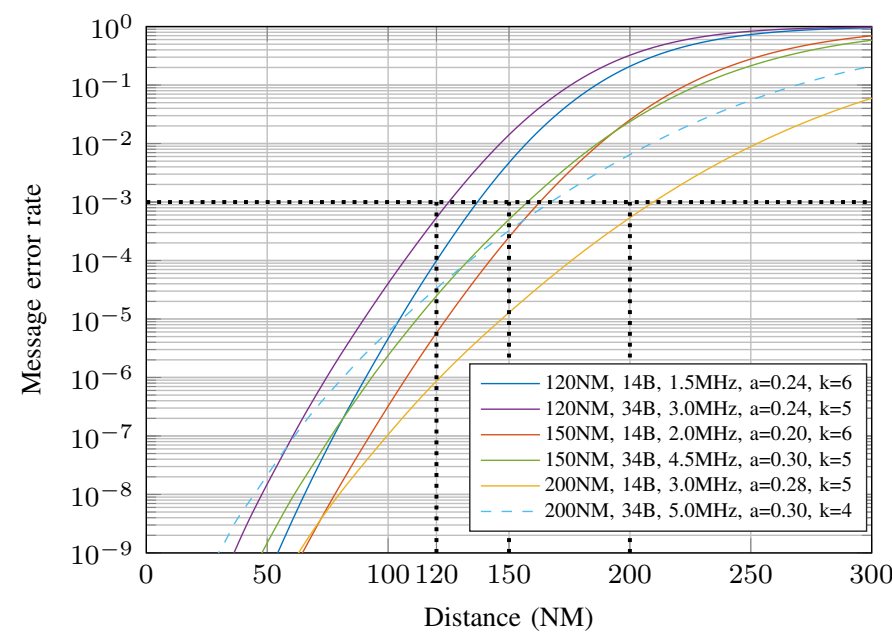

Fig. 5. MER of the ALOHA-based A2A data link for a given distance between the transmitter and receiver. Performance obtained for $\rho=0.0055$ aircraft $/ \mathrm{NM}^{2}$ and $N_{s c}=1$. The black dotted lines highlight the maximum allowed MER and the nominal communication ranges.

is achieved or the maximum considered bandwidth, i.e., 5 $\mathrm{MHz}$, is reached. For each particular case, $a$ and $k$ are varied within a certain range, specifically $a=[0.1: 0.02: 1]$ and $k=[1: 1: 10]$, until the minimum MER at $R_{c}$ is achieved. We show in Fig. 5 the obtained optimum performance for each combination of $R_{c}$ and $L$ in terms of the MER, computed using (9), against the distance between the transmitter and the receiver. As a general convention for the paper, we use lines with different colors to distinguish the different combinations of $R_{c}$ and $L$. In addition, the combinations fulfilling the required performance are depicted using continuous lines, whilst those not achieving it are plotted using dashed lines. The maximum allowed MER and the nominal communication ranges are highlighted with dotted black lines. One can see that, in order to achieve a MER lower than $10^{-3}$ at each nominal communications range, an ALOHA-based A2A data link would need a bandwidth of $1.5 \mathrm{MHz}, 2 \mathrm{MHz}$, and 3 $\mathrm{MHz}$, for a nominal communications range of $120 \mathrm{NM}, 150$ $\mathrm{NM}$, and $200 \mathrm{NM}$, respectively, and $L=14$ information bytes. Likewise, conveying 34 information bytes per message up to a nominal distance of $120 \mathrm{NM}$ and $150 \mathrm{NM}$ with the minimum required performance entails a minimum bandwidth of $3 \mathrm{MHz}$ and $4.5 \mathrm{MHz}$, respectively. Note that a nominal communications range of $200 \mathrm{NM}$ is not possible with the desired performance when transmitting 34 information bytes per message with a bandwidth $B W \leq 5 \mathrm{MHz}$.

We repeat the previous simulations for a total bandwidth $B W$ split into $N_{s c}$ sub-channels of $0.5 \mathrm{MHz}$ each. The obtained performance is, in any considered case, worse than the one obtained for a single channel, specially for lower distances between the transmitter and the receiver. This is due to the fact that the message duration is increased because of the lower bandwidth used per message, which increases the probability of an aircraft to interfere its receptions with its 


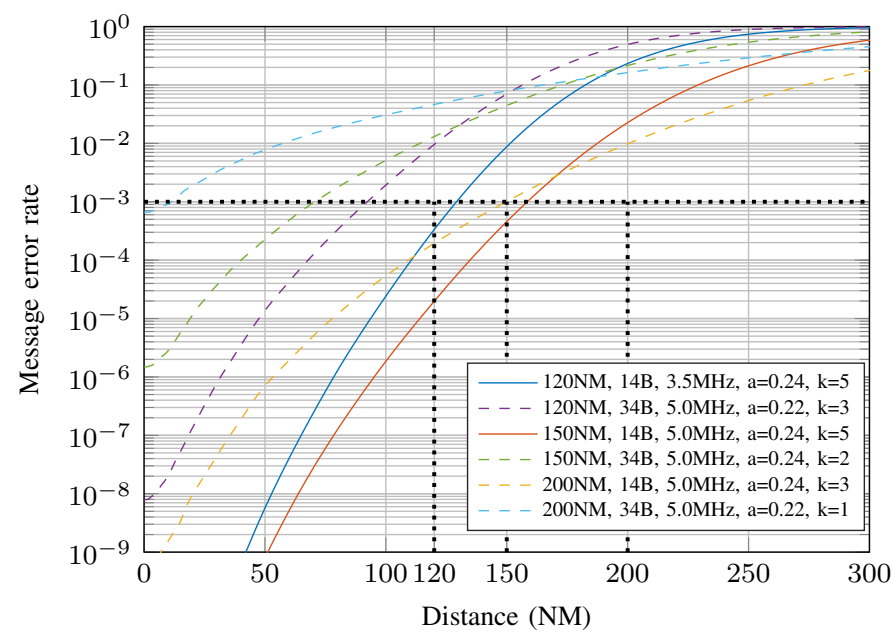

Fig. 6. MER of the ALOHA-based A2A data link for a given distance between the transmitter and receiver. Performance obtained for $\rho=0.0150$ aircraft/NM ${ }^{2}$ and $N_{s c}=1$.

own transmissions, as shown in (7). However, the obtained minimum bandwidth for each considered case is the same as the one shown in Fig. 5 for a single channel. Consequently, we do not show the results here for simplicity.

We now consider the higher aircraft density and repeat the simulations for $N_{s c}=1$, obtaining the performance shown in Fig. 6. One can see that an ALOHA-based A2A data link could only achieve the desired performance in such a dense air traffic for a communications range of either $120 \mathrm{NM}$ or $150 \mathrm{NM}$ by using a bandwidth of $3.5 \mathrm{MHz}$ and $5 \mathrm{MHz}$, respectively, and transmitting only 14 information bytes per message. Any other considered case does not achieve the desired performance for a bandwidth $B W \leq 5 \mathrm{MHz}$.

Simulations are repeated for a total bandwidth $B W$ split into $N_{s c}$ sub-channels of $0.5 \mathrm{MHz}$ each. The obtained results, which are again not depicted here because the same required bandwidths are obtained as those shown in Fig. 6 for a single channel, indicate again that dividing the total bandwidth into sub-channels is detrimental for an ALOHA-based A2A data link, as the performance is worse in any considered case.

\section{B. Results for the STDMA-Based A2A Data Link}

In comparison to the simulations run for ALOHA, the model of the STDMA-based A2A data link requires an extremely high computational effort to simulate each considered case. Consequently, we do not optimize the value of $a$ for each particular case but only for each combination of $R_{c}$ and $L$ when a bandwidth of $0.5 \mathrm{MHz}$ and an aircraft density of 0.0055 aircraft/ $\mathrm{NM}^{2}$ are used. For STDMA, we do not consider the transmission of replicas, i.e., $k=1$. Evaluating the aforementioned cases for $a=[0.1: 0.1: 0.9]$, the resulting optimum values of $a$ are $0.3,0.3$, and 0.4 , for 14 information bytes transmitted per message and a communications range of $120 \mathrm{NM}, 150 \mathrm{NM}$, and $200 \mathrm{NM}$, respectively. For 34 information bytes transmitted per message, the optimum values of $a$ are $0.6,0.6$, and 0.8 , for a communications range of

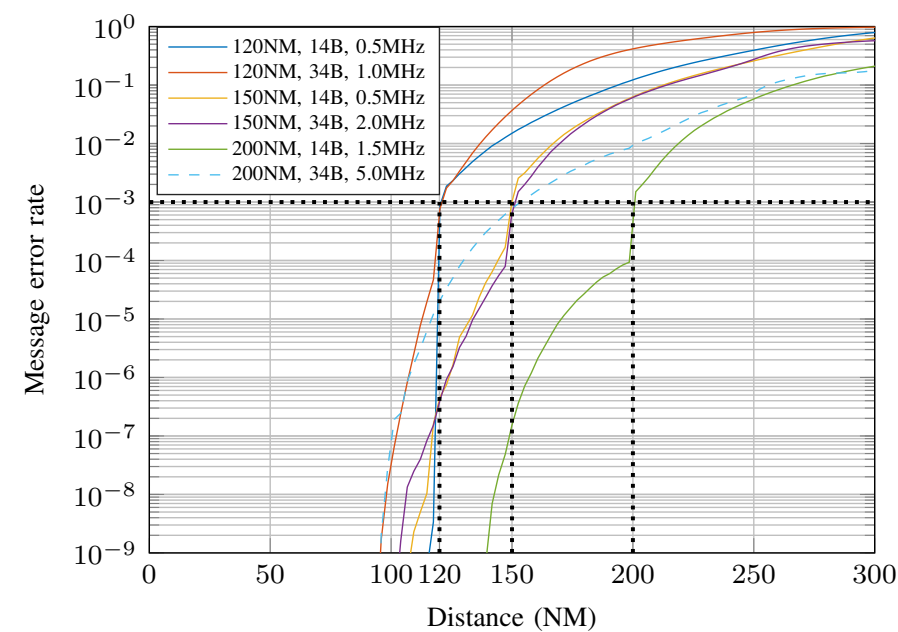

Fig. 7. MER of the STDMA-based A2A data link for a given distance between the transmitter and receiver. Performance obtained for $\rho=0.0055$ aircraft/NM ${ }^{2}$ and $N_{s c}=1$.

$120 \mathrm{NM}, 150 \mathrm{NM}$, and $200 \mathrm{NM}$, respectively. We use these values throughout our analysis for each combination of $R_{c}$ and $L$ despite the increase in the bandwidth, aircraft density, or number of frequency channels.

We now simulate the STDMA-based A2A data link for each combination of $R_{c}$ and $L$, increasing the used bandwidth from $0.5 \mathrm{MHz}$ in steps of $0.5 \mathrm{MHz}$ until a MER lower than $10^{-3}$ is achieved at the communications range. Again, the maximum considered bandwidth is limited to $5 \mathrm{MHz}$. The obtained results for $\rho=0.0055$ aircraft/NM $\mathrm{NM}^{2}$ and $N_{s c}=1$ are shown in Fig. 7. One can see that the required performance is achieved with a bandwidth of $0.5 \mathrm{MHz}, 0.5 \mathrm{MHz}$, and $1.5 \mathrm{MHz}$, when transmitting 14 information bytes per message at a communications range of $120 \mathrm{NM}, 150 \mathrm{NM}$, and $200 \mathrm{NM}$, respectively. For the transmission of 34 information bytes per message, both communications ranges of $120 \mathrm{NM}$ and $150 \mathrm{NM}$ can be achieved with the desired performance using a bandwidth of $1 \mathrm{MHz}$ and $2 \mathrm{MHz}$, respectively. However, transmitting 34 information bytes per message up to a communications range of $200 \mathrm{NM}$ cannot be achieved with the desired performance and a bandwidth limited to $5 \mathrm{MHz}$. As expected, broadcasting larger data packets and considering longer communication ranges lead to longer time slots and, consequently, to less time slots per frame, increasing the probability that an aircraft will knowingly have to transmit in a time slot reserved by other aircraft and increasing the probability of collisions. In addition, even though there are more users than slots in the system, the scheduling algorithm allows each aircraft to choose the most suitable slot. The effect of this scheduling in congested scenarios can be clearly seen in Fig. 7 for 200 NM and 14 information bytes, which shows a MER that decreases softly from the communications range towards lower distances.

Let us now consider that the total bandwidth is split into $N_{s c}$ frequency sub-channels of $0.5 \mathrm{MHz}$ each. The obtained performance and the minimum required bandwidth for each 


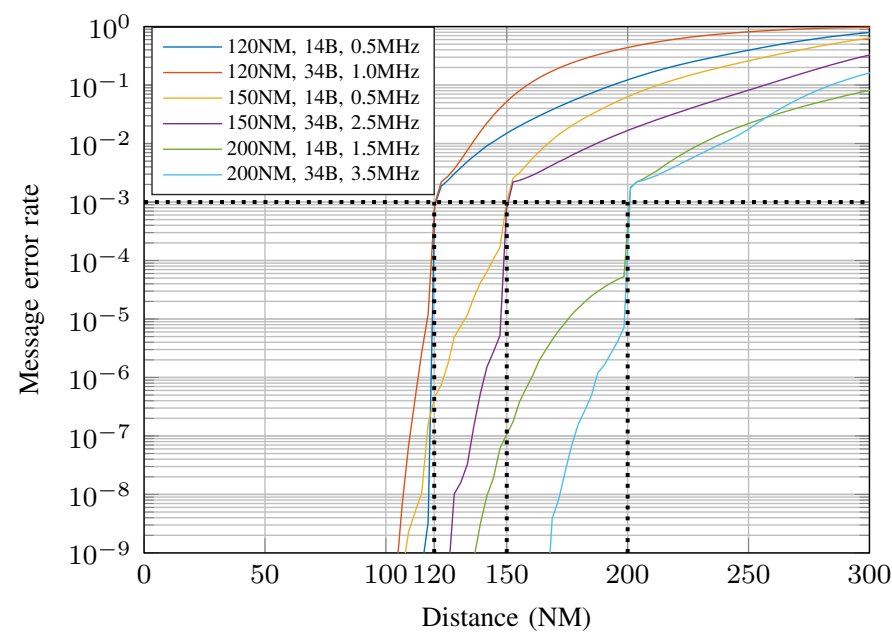

Fig. 8. MER of the STDMA-based A2A data link for a given distance between the transmitter and receiver. Performance obtained for $\rho=0.0055$ aircraft $/ \mathrm{NM}^{2}$ and $N_{s c}=\frac{B W}{0.5 \mathrm{MHz}}$ frequency sub-channels.

considered case are shown in Fig. 8. In comparison to the results depicted in Fig. 7 for a single channel, one can see that the same minimum required bandwidth is obtained for $R_{c}=$ $120 \mathrm{NM}$ and both 14 and 34 information bytes per message, for $R_{c}=150 \mathrm{NM}$ and 14 information bytes, and for $R_{c}=$ $200 \mathrm{NM}$ and 14 information bytes. Splitting the bandwidth into sub-channels of $0.5 \mathrm{MHz}$ each is, however, detrimental for $R_{c}=150 \mathrm{NM}$ and 34 information bytes, as the required bandwidth is increased by $0.5 \mathrm{MHz}$. Nevertheless, the benefit of using multiple sub-channels of $0.5 \mathrm{MHz}$ can be clearly seen in Fig. 8 for $R_{c}=200 \mathrm{NM}$ and 34 information bytes. Whilst this case could not be supported with a single channel and a bandwidth $B W \leq 5 \mathrm{MHz}$, the required performance can be achieved with only $3.5 \mathrm{MHz}$ if multiple sub-channels of 0.5 $\mathrm{MHz}$ are employed.

Then, we evaluate the performance for the higher aircraft density, i.e., $\rho=0.0150$ aircraft/ $\mathrm{NM}^{2}$. The obtained performance is shown in Fig. 9 and Fig. 10 for a single channel and $N_{s c}$ frequency sub-channels of $0.5 \mathrm{MHz}$ each, respectively. As it can be seen, the minimum required performance can be achieved by the STDMA-based A2A link for a communications range of $120 \mathrm{NM}$ and 14 information bytes per message with only $1 \mathrm{MHz}$ bandwidth if one channel is considered, and $2 \mathrm{MHz}$ if multiple sub-channels are considered. For the same communications range, transmitting 34 information bytes per message requires a bandwidth of $3.5 \mathrm{MHz}$ in one single channel or $3 \mathrm{MHz}$ in multiple sub-channels. For a communications range of $150 \mathrm{MHz}$, however, the required performance can only be achieved for 14 information bytes per message and a single channel of $1.5 \mathrm{MHz}$. In any other case with a communications range of $150 \mathrm{NM}$, a bandwidth of $5 \mathrm{MHz}$ would not suffice to achieve the desired performance. Finally, the required performance can be achieved with a communications range of $200 \mathrm{NM}$ if 14 information bytes are transmitted per message and either one single channel of

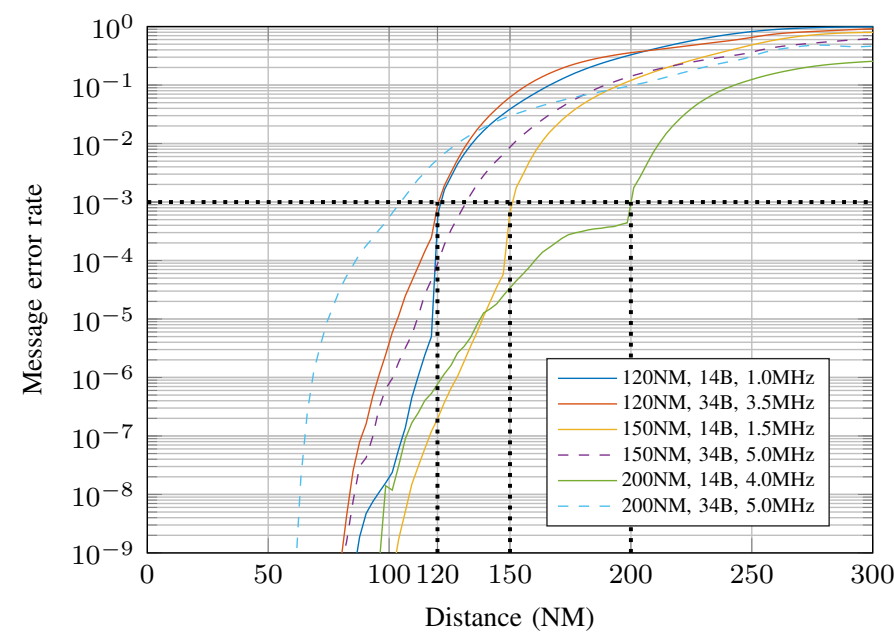

Fig. 9. MER of the STDMA-based A2A data link for a given distance between the transmitter and receiver. Performance obtained for $\rho=0.0150$ aircraft/NM ${ }^{2}$ and $N_{s c}=1$.

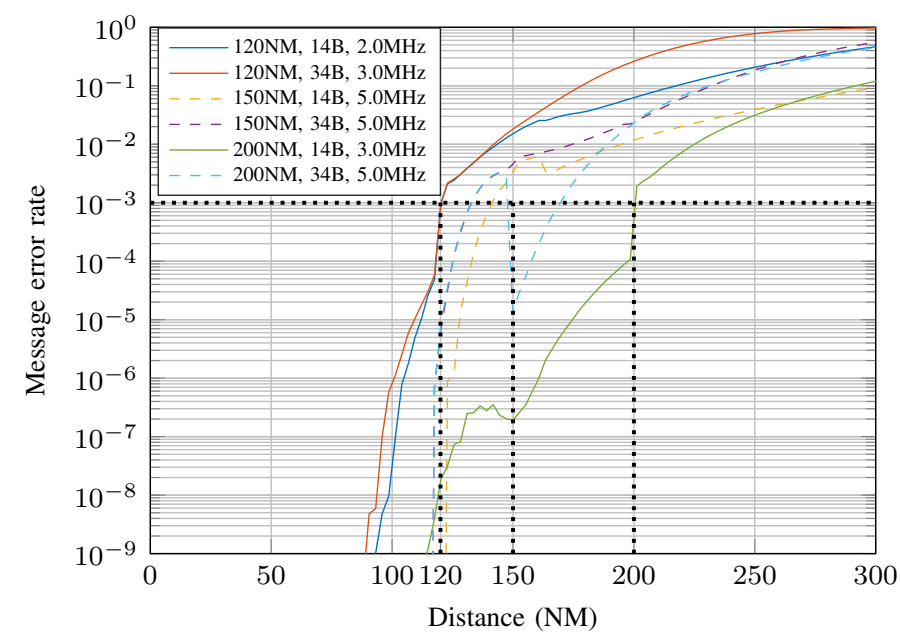

Fig. 10. MER of the STDMA-based A2A data link for a given distance between the transmitter and receiver. Performance obtained for $\rho=0.0150$ aircraft $/ \mathrm{NM}^{2}$ and $N s c=\frac{B W}{0.5 \mathrm{MHz}}$ frequency sub-channels.

$4 \mathrm{MHz}$ or 6 sub-channels with a total bandwidth of $3 \mathrm{MHz}$ are employed. However, the required performance can not be achieved for $R_{c}=200 \mathrm{NM}$ if messages containing 34 information bytes are broadcast in such a dense air traffic, even when sub-channels are employed.

Note that, illogically, broadcasts of 14 information bytes can be supported for $R_{c}=200 \mathrm{NM}$ but not for $R_{c}=150 \mathrm{NM}$ when sub-channels are used, as shown in Fig. 10. We attribute this to either the usage of sub-channels, which is beneficial or detrimental depending on each specific case, or the simple criterion used for the scheduling.

Until now, we have assumed that all aircraft are capable of listening to all reservations up to $R_{l o s}$, which is a necessary initial assumption but leads to optimistic results. In order to assess how a realistic performance impacts the scheduling 


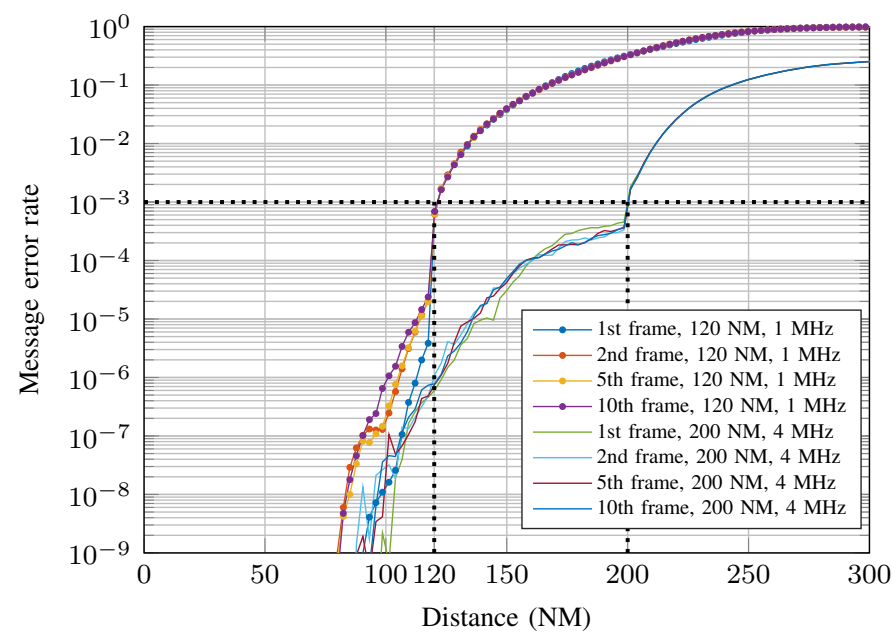

Fig. 11. MER of the STDMA-based A2A data link for a given distance between the transmitter and receiver considering a realistic scheduling. Performance obtained for $\rho=0.0150$ aircraft/NM ${ }^{2}, N s c=1$, and 14 information bytes transmitted per message.

process and consequently the performance of the STDMAbased A2A data link, we simulate some of the considered cases for 10 consecutive frames as discussed in Section IV-B2. Note that this process requires an extremely high computational effort and consequently it has only been performed for two representative cases.

We obtain the results of the STDMA-based A2A data link with memory for the higher aircraft density, one single channel, 14 information bytes transmitted per message, and two different communication ranges; 120 NM and $200 \mathrm{NM}$. For each case, we use the minimum required bandwidth that was obtained before for the optimum scheduling, i.e., $1 \mathrm{MHz}$ for $120 \mathrm{NM}$ and $4 \mathrm{MHz}$ for $200 \mathrm{NM}$. Results are shown jointly in Fig. 11 for the first, second, fifth, and tenth frame of each case. From the obtained results, one can see a soft degradation in the performance caused by the realistic selforganization. However, this degradation does not strongly affect the STDMA-based A2A data link as the required performance is still achieved with the same bandwidth. In addition, it is to be noted that the performance stabilizes very quickly after the first frame.

\section{CONCLUSION}

In this paper, we contribute to the development of the LDACS A2A data link by assessing the performance of an A2A data link based on two different medium-access protocols; ALOHA with and without diversity, and STDMA. The performance is obtained by simulating the defined models of the ALOHA-based A2A data link and the STDMA-based A2A data link for different design parameters, requirements, and air traffic conditions. The obtained results show that the STDMAbased A2A data link performs better than the ALOHA-based A2A data link in most of the considered cases, given than the former requires a lower bandwidth than the latter to achieve the desired performance. In addition, splitting the available bandwidth of the system into sub-channels of $0.5 \mathrm{MHz}$, which is expected to be needed for the LDACS A2A, is significantly detrimental for an ALOHA-based A2A data link. By contrast, it can be very beneficial for the STDMA-based A2A data link, specially for the most challenging scenarios. For these reasons, we conclude that STDMA is a better candidate than ALOHA with or without diversity for the medium-access control of the LDACS A2A.

\section{REFERENCES}

[1] EUROCONTROL/FAA Future Communications Study Operational Concepts and Requirements Team, "Communications operating concept and requirements for the future radio system (COCR), ver. 2," 2007.

[2] T. Graupl, N. Schneckenburger, T. Jost, M. Schnell, A. Filip, M. A. Bellido-Manganell, D. M. Mielke, N. Maurer, R. Kumar, O. Osechas, and G. Battista, "L-band digital aeronautical communications system (LDACS) flight trials in the national German project MICONAV," in Proc. IEEE Integr. Commun. Navigat. Surveillance Conf., Herndon, VA, USA, 2018, pp. 4A2-1-4A2-7.

[3] M. Sajatovic, B. Haindl, U. Epple, and T. Gräupl, "Updated LDACS1 system specification,' SESAR Joint Undertaking Report EWA04-1-T2D1, 2011

[4] M. Schnell, U. Epple, D. Shutin, and N. Schneckenburger, "LDACS: Future aeronautical communications for air-traffic management," IEEE Communications Magazine, vol. 52, no. 5, pp. 104-110, May 2014.

[5] N. Abramson, "THE ALOHA SYSTEM: Another alternative for computer communications," in Proc. Fall Joint Comp. Conf., pp. 281-285, 1970.

[6] L. G. Roberts, "ALOHA packet system with and without slots and capture," ACM SIGCOMM Computer Communication Review, vol. 5, no. 2, pp. 28-42, 1975.

[7] G. Choudhury and S. Rappaport, "Diversity aloha-a random access scheme for satellite communications," IEEE Transactions on Communications, vol. 31, no. 3, pp. 450-457, 1983

[8] G. Gallinaro, F. Di Cecca, M.-A. Marchitti, R. de Gaudenzi, and O. Del Rio Herrero, "Enhanced spread spectrum aloha system level performance assessment," International Journal of Satellite Communications and Networking, vol. 32, no. 6, pp. 485-503, 2014.

[9] Minimum Operational Performance Standards for $1090 \mathrm{MHz}$ Extended Squitter Automatic Dependent Surveillance - Broadcast (ADS-B) and Traffic Information Services - Broadcast (TIS-B). RTCA, Inc., Washington, DC, USA, RTCA DO-260B, 2019.

[10] H. Lans, "Position indicating system," U.S. Patent 5506 587, 1996.

[11] Technical characteristics for an automatic identification system using time-division multiple access in the VHF maritime mobile band. International Telecommunication Union, Recommendation ITU-R M.1371-4, 2010.

[12] Manual on VHF Digital Link (VDL) Mode 4. International Civil Aviation Organization, 1st edition, 2004.

[13] FlightRadar24 homepage: https://www.flightradar24.com/.

[14] The OpenSky Network homepage: https://opensky-network.org/.

[15] T. Graupl, "FACTS2: A service oriented simulation framework for aeronautical communication system evaluation," in Proc. IEEE/AIAA 35th Digit. Avionics Syst. Conf., Sacramento, CA, USA, 2016, pp. 1-7.

[16] _ "Validating the FACTS2 air traffic simulation framework," in Proc. IEEE Integr. Commun. Navigat. Surveillance Conf., Herndon, VA, USA, 2017, pp. 1D4-1-1D4-8.

[17] — - "FACTS2: Extended simulation framework for ATM communication demand analysis of Europe," in Proc. IEEE/AIAA 36th Digit. Avionics Syst. Conf., St. Petersburg, FL, USA, 2017.

[18] N. Franzen, "Spectrally efficient beaconing for aeronautical applications," Ph.D. dissertation, Technical University Darmstadt, July 2015.

[19] L. M. Schalk, "Techniques for improving the cooperative traffic conflict detection among drones," in Proc. IEEE 89th Vehic. Techn. Conf. (VTC2019-Spring), Kuala Lumpur, Malaysia, Malaysia, May 2019.

[20] M. Abramowitz and I. A. Stegun, Handbook of mathematical functions: with formulas, graphs, and mathematical tables. Courier Corporation, 1965, vol. 55 .

[21] Minimum Aviation System Performance Standards for Automatic Dependent Surveillance Broadcast (ADS-B). RTCA, Inc., Washington, DC, USA, RTCA DO-242A, June 2002. 